\title{
Temporal moments of a tracer pulse in a perfectly parallel flow system
}

Advances in Water Resources 2003, v.26, p.599-607

\author{
W. E. Bardsley \\ Department of Earth \& Ocean Sciences \\ University of Waikato \\ Private Bag 3105 \\ Hamilton \\ New Zealand
}

e-mail:web@waikato.ac.nz 


\begin{abstract}
Perfectly parallel groundwater transport models partition water flow into isolated one-dimensional stream tubes which maintain total spatial correlation of all properties in the direction of flow. The case is considered of the temporal moments of a conservative tracer pulse released simultaneously into $N$ stream tubes with arbitrarily different advective-dispersive transport and steady flow speeds in each of the stream tubes. No assumptions are made about the form of the individual stream tube arrival-time distributions or about the nature of the between-stream tube variation of hydraulic conductivity and flow speeds. The tracer arrival-time distribution $g(t, x)$ is an $N$-component finitemixture distribution, with the mean and variance of each component distribution increasing in proportion to tracer travel distance $x$. By utilising moment relations of finite mixture distributions, it is shown (to $r=4$ ) that the $r$ th central moment of $g(t, x)$ is an $r$ th order polynomial function of $x$ or $\phi$, where $\phi$ is mean arrival time. In particular, the variance of $g(t, x)$ is a positive quadratic function of $x$ or $\phi$. This generalises the well-known quadratic variance increase for purely advective flow in parallel flow systems and allows a simple means of regression estimation of the large-distance coefficient of variation of $g(t, x)$. The polynomial central moment relation extends to the purely advective transport case which arises as a large-distance limit of advective-dispersive transport in parallel flow models. The associated limit $g(t, x)$ distributions are of $N$-modal form and maintain constant shapes independent of travel distance. The finite-mixture framework for moment evaluation is also a potentially useful device for forecasting $g(t, x)$ distributions, which may include multimodal forms. A synthetic example illustrates $g(t, x)$ forecasting using a mixture of normal distributions.
\end{abstract}

Key words: Stratified aquifer; Moments; Advection-dispersion; Mixed distribution 


\section{Introduction}

Parallel flow models provide a convenient representation of one-dimensional tracer dispersion by groundwater movement in aquifers with significant stratification, or with preferential flow paths in the direction of flow. The concept of parallel groundwater flow systems dates at least to the work of Marle [19] and Mercado [21], the latter utilising a perfectly stratified aquifer model as a visualisation of purely advective tracer dispersion. Parallel flow models have also been used for some time as special cases of discrete fracture networks [24, 30]. More recently, parallel models have been employed as multiple stream tube models for the theoretical study of reactive processes in heterogeneous aquifers [5], for developing crack-flow dispersion models for radioactive wastes [4, 17], in bioremediation studies [25], and as a framework for evaluating analytical approximations in groundwater flow theory [18].

The individual flow tubes in the parallel flow model have been referred to as "channels" for fractureflow and "layers" in the context of perfectly stratified aquifer models. The more general term "stream tube" will be used here in recognition of the essential one-dimensional nature of parallel groundwater flow systems. The term "perfectly parallel" is used here to emphasise that no exchange takes place between the individual stream tubes.

Tracer transport in parallel systems provides a non-diffusive mechanism contributing to dispersion $[21,20,12,28,13,15,35]$. This stream-tube dispersion effect has been referred to as a "convective dispersive" process $[33,16]$, causing tracer arrival-time variance to increase as a quadratic function of travel distance for advective flow. This quadratic relation has been derived under specific conditions with respect to tracer transport in both perfectly parallel systems and imperfectly parallel systems in the vicinity of the tracer origin $[21,30,24 ; 32,22,11,33]$.

The use of variances and other distribution moment measures in dispersion studies serve the useful purpose of avoiding detailed physical models requiring full distribution parameterisation [38]. However, past investigations dealing with moments and other aspects of parallel or partially-parallel flow systems tend to have been restricted to special cases. For example, specification of normal distributions of flow velocities between the stream tubes [21], pure advective flow within the stream tubes [21, 30, 24], normal or lognormal arrival times [11, 33], lognormal distributions of hydraulic conductivities [7], and other various specified forms of hydraulic conductivity distribution [8, 13]. 
There appears to have been no derivation to date of general temporal moment expressions for parallel flow systems, where the expressions are both explicit and sufficiently general to allow arbitrarily different advective-dispersive transport in the different stream tubes. Currently available temporal moment expressions tend to be complex and sometimes require the specification of particular subexpressions $[32,22,14]$.

The purpose of this paper is to show that for perfectly parallel systems with steady flow, the arrivaltime moments are simple polynomial functions of tracer travel distance $x$, all other terms being constants. Specifically, it is shown, to $r=4$, that the $r$ th temporal central moment of a conservative tracer pulse is an $r$ th order polynomial function of tracer travel distance alone. This polynomial relation holds for both advective-dispersive stream tube transport and pure advective transport. The well known quadratic increase of arrival-time variance for pure advective flow in parallel systems is therefore a special case of a more general relation for parallel flow models. The polynomial relation is robust to the extent that each stream tube is permitted arbitrary independent advective-dispersive transport and the arrival-time distributions can be of arbitrary multimodal form. Also, there is no requirement for any specific distribution of hydraulic conductivity and flow speeds between the stream tubes.

The focus here on temporal rather than spatial moments was made because arrival-time data have application to forecasting environmental impacts of migrating contaminant plumes and have sometimes been incorporated in licensing criteria [23,9]. Also, arrival-time data have the advantage of being relatively easy to collect at a few specific observation points in field or laboratory column investigations $[31,36,37]$.

\section{The perfectly parallel flow model}

The parallel flow model utilised here for temporal moment derivations follows along the lines of the advective-dispersive multiple stream tube model described by [5]. As illustrated in Fig. 1, the utilised model is a linear system comprised of an ensemble of an arbitrary number of $(N \geq 2)$ independent one-dimensional stream tubes of equal length $x$. Each stream tube has arbitrary and independent hydraulic properties, but with perfect spatial correlation of all properties along each stream tube. This 
configuration has been referred to as the "basic" parallel flow unit in the context of a fracture network [30].

The stream tubes originate from a conceptual mixing plane perpendicular to the stream tubes. This plane generates a brief pulse of conservative tracer particles simultaneously into all stream tubes, with equal numbers of particles entering each stream tube at time zero. All the tracer particles are deemed identical, so partitioning of the tracer pulse into the $N$ stream tubes can be viewed equivalently in terms of equal particle numbers or equal mass fractions.

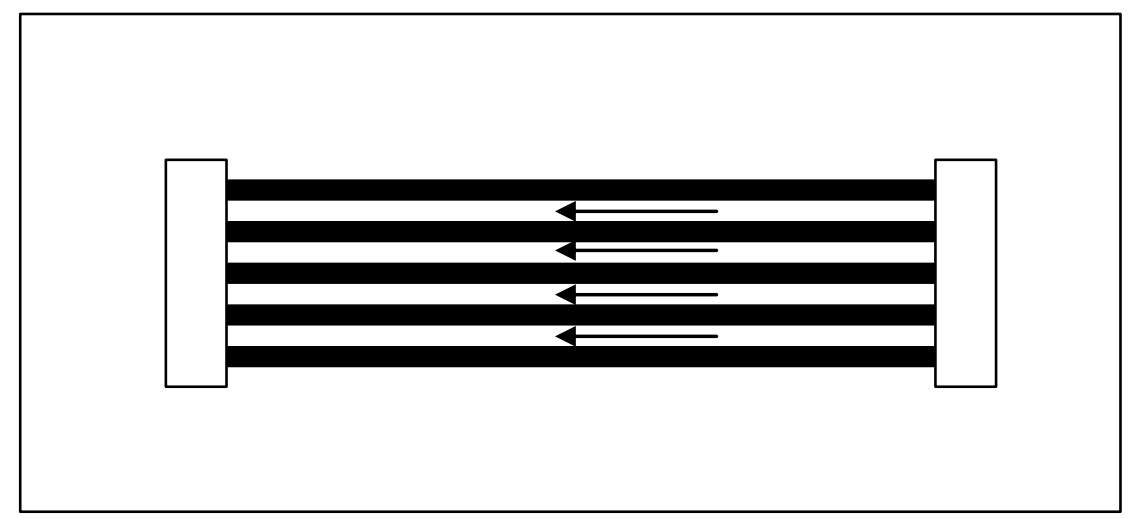

Fig. 1 Schematic of parallel flow model, after [30].

It may seem unnecessarily restrictive to require exactly equal numbers of particles to enter each stream tube. However, no loss of generality is involved because for any unequal parallel flow system there are mathematically equivalent parallel systems with equal numbers of tracer particles per stream tube. For example, suppose $N=2$ in an unequal model, with one third of the total tracer particle pulse entering one stream tube and two-thirds entering the other. An equivalent equal model could then be set up with $N=3$, where the dominant stream tube is replaced by two stream tubes each transporting $1 / 3$ of the total tracer pulse.

Within each stream tube the tracer particles undergo arbitrarily different and independent advectivedispersive transport as a consequence of (different) steady one-dimensional flow per stream tube. That is, the tracer arrival-time mean and variance always increase linearly with travel distance in each stream tube, but the rates of increase are arbitrarily different between stream tubes. Following 
[20] and [29], the advective-dispersive process is viewed here as Brownian motion with drift, where the Brownian motion is taken to incorporate all hydrodynamic dispersion effects

The stream tubes terminate at a conceptual outflow mixing surface, which may be an observation well, at distance $x$ from the input plane. Tracer concentrations are visualised as being recorded over time at the outflow surface where the tracer contributions of all stream tubes are mixed. This tracer concentration time series defines the arrival-time distribution $g(t, x)$, for travel distance $x$. Arrivaltime distributions of this type are flux-weighted by definition [7]. That is, $g(t, x)$ is a flux-weighted integration over all stream tubes for any given $t$.

Thus defined, the arrival-time distribution $g(t, x)$ will evidently be an $N$-component finite-mixture probability density function with equal weight $N^{-1}$ on each of the component distributions, symbolised as:

$$
g(t, x)=N^{-1} \sum_{i=1}^{N} f_{i}(t, x)
$$

The component $f_{i}(t, x)$ distributions here are the arrival-time distributions specific to the respective stream tubes. The required temporal moment expressions can therefore be obtained as the moments of the finite mixture distribution given by Eq. (1), utilising the temporal moments of the component distributions. This a more direct approach than deriving temporal moments via the Aris method [1] from a specified set of boundary conditions. The finite mixture approach was also utilised by [30] in an advection flow parallel model with unequal component distribution weights.

The pure advection special case of $g(t, x)$ arises when all the $f_{i}(t, x)$ variances remain zero. The shapes of the $g(t, x)$ distribution then remain unchanged with travel distance $x$. This is simply a consequence of the progressive linear increase in the time-separation between the consecutive component pulses from the different stream tubes transporting the brief tracer pulse. 


\section{Derivations}

The cumulant ratios of the component $f_{i}(t, x)$ distributions will remain constant and independent of $x$. This is a basic property of the cumulants of sums of independent and identically distributed random variables. For example, distribution mean and variance are both cumulants so doubling $x$ doubles both the mean and variance of the $f_{i}(t, x)$ distributions, while their respective mean/variance ratios remaining unchanged. A formal definition of distribution cumulants can be found in statistical texts - see also [30]. A listing of cumulant expressions in terms of distribution moments is given by $[34$, p.87-88].

Omitting the $i$ subscript for now, define the set of cumulant ratios $Z_{r}$ of a single $f(t, x)$ distribution as:

$$
Z_{r}=\kappa_{r} / \kappa_{1}=\kappa_{r} / \mu_{1}^{\prime} \quad r \geq 2
$$

where $\mu_{1}^{\prime}$ is the first moment about zero (distribution mean), and $\kappa_{r}$ is the $r$ th cumulant of the component distribution concerned. Because the $Z_{r}$ values are constant cumulant ratios, they are independent of $\mu_{1}^{\prime}$ and hence are independent of tracer travel distance $x$.

In general, any distribution's $r$ th moment about zero $\left(\mu_{r}^{\prime}\right)$ can be written as a function of the distribution cumulants. For the second, third, and fourth moments about zero, these expressions are, respectively, [34, p.86]:

$$
\begin{aligned}
& \mu_{2}^{\prime}=\kappa_{2}+\kappa_{1}^{2} \\
& \mu_{3}^{\prime}=\kappa_{3}+3 \kappa_{2} \kappa_{1}+\kappa_{1}^{3} \\
& \mu_{4}^{\prime}=\kappa_{4}+4 \kappa_{3} \kappa_{1}+3 \kappa_{2}^{2}+6 \kappa_{2} \kappa_{1}^{2}+\kappa_{1}^{4}
\end{aligned}
$$


The corresponding expressions in terms of $Z_{r}$ and $\mu_{1}^{\prime}$ are obtained from Eqs. (3), (4), and (5) by substituting $\kappa_{r}$ with $Z_{r} \mu_{1}^{\prime}$ (for $r \geq 2$ ), and substituting $\kappa_{1}$ with $\mu_{1}^{\prime}$, giving:

$$
\begin{aligned}
& \mu_{2}^{\prime}=Z_{2} \mu_{1}^{\prime}+\mu_{1}^{\prime 2} \\
& \mu_{3}^{\prime}=Z_{3} \mu_{1}^{\prime}+3 Z_{2} \mu_{1}^{\prime 2}+\mu_{1}^{\prime 3} \\
& \mu_{4}^{\prime}=Z_{4} \mu_{1}^{\prime}+\left(4 Z_{3}+3 Z_{2}^{2}\right) \mu_{1}^{\prime 2}+6 Z_{2} \mu_{1}^{\prime 3}+\mu_{1}^{\prime 4}
\end{aligned}
$$

In the context of a given $f(t, x)$ distribution, it is evident from Eqs. (6), (7), and (8) that the distribution moments about zero are evidently polynomial functions of $\mu_{1}^{\prime}$, and therefore of $x$.

Turning now to the parallel flow system as a whole and reintroducing the $i$ subscript, define $\tau$ and $\tau_{i}$ respectively as random variables generated from $g(t, x)$ and $f_{i}(t, x)$, and define:

$$
\begin{aligned}
& \phi=E(\tau) \\
& \phi \alpha_{i}=\mu_{1 i}^{\prime}=E\left(\tau_{i}\right)
\end{aligned}
$$

where the $\alpha_{i}$ values in Eq. (10) are constants independent of $x$. This constancy of the $\alpha_{i}$ values arises because each stream tube has its own constant flow speed, causing each $E\left(\tau_{i}\right)$ to remain in constant ratio with $E(\tau)$.

Define $\mu_{r i}^{\prime}, \mu_{r i}$, and $\kappa_{r_{i}}$, respectively, as the $r$ th moment about zero, $r$ th central moment, and $r$ th cumulant, of $f_{i}(t, x)$. Similarly, $\mu_{r}^{\prime}$ and $\mu_{r}$ are the $r$ th moment about zero and $r$ th central moment of $g(t, x)$. The $f_{i}(t, x)$ and $g(t, x)$ moments have been referred to respectively as "local temporal moments" and "integrated temporal moments" [6]. The symbolism adopted in this paper serves to make the same distinction. 
As noted in Eq. (2), the cumulant ratios specific to each $f_{i}(t, x)$ are defined:

$$
Z_{r i}=\kappa_{r i} / \mu_{1 i}^{\prime} \quad r \geq 2
$$

and the $r$ th moment about zero of $g(t, x)$ is the mean of the component distribution moments about zero:

$\mu_{r}^{\prime}=N^{-1} \sum_{i=1}^{N} \mu_{r i}^{\prime}$

It follows from Eqs. (6)-(8) that the corresponding moments of $g(t, x)$ about zero can be expressed (omitting the summation range for brevity):

$$
\begin{aligned}
& \mu_{2}^{\prime}=N^{-1} \phi \sum Z_{2 i} \alpha_{i}+\phi^{2} \sum \alpha_{i}^{2} \\
& \mu_{3}^{\prime}=N^{-1} \phi \sum Z_{3 i} \alpha_{i}+3 \phi^{2} \sum Z_{2 i} \alpha_{i}^{2}+\phi^{3} \sum \alpha_{i}^{3} \\
& \mu_{4}^{\prime}=N^{-1} \phi \sum Z_{4 i} \alpha_{i}+\phi^{2} \sum\left(4 Z_{3 i}+3 Z_{2 i}^{2}\right) \alpha_{i}^{2}+6 \phi^{3} \sum Z_{2 i} \alpha_{i}^{3}+\phi^{4} \sum \alpha_{i}^{4}
\end{aligned}
$$

The corresponding central moments of $g(t, x)$ can now be obtained by substituting the $g(t, x)$ moments (13)-(15) into the standard statistical expressions giving distribution central moments as functions of moments about zero. This yields the central moments of $g(t, x)$ as simple polynomial functions of mean travel time $\phi$ :

$$
\begin{aligned}
\mu_{2}= & \phi N^{-1} \sum Z_{2 i} \alpha_{i}+\phi^{2} N^{-1} \sum \alpha_{i}^{2}-1 \\
\mu_{3}= & \phi N^{-1} \sum Z_{3 i} \alpha_{i}+3 \phi^{2} N^{-1} \sum Z_{2 i} \alpha_{i}^{2}-\sum Z_{2 i} \alpha_{i}+\phi^{3} N^{-1} \sum \alpha_{i}^{3}-3 \sum \alpha_{i}^{2}+2 N \\
\mu_{4}= & \phi N^{-1} \sum Z_{4 i} \alpha_{i}+\phi^{2} N^{-1} 4 \sum Z_{3 i} \alpha_{i}^{2}+3 \sum Z_{2 i}^{2} \alpha_{i}^{2}-4 \sum Z_{3 i} \alpha_{i} \\
& +6 \phi^{3} N^{-1} \sum Z_{2 i} \alpha_{i}^{3}-2 \sum Z_{2 i} \alpha_{i}^{2}+\sum Z_{2 i} \alpha_{i}+\phi^{4} N^{-1} \sum \alpha_{i}^{4}-4 \sum \alpha_{i}^{3}+6 \sum \alpha_{i}^{2}-3 N
\end{aligned}
$$


where Eqs. (16), (17), and (18) are respectively the second, third and fourth central moment of $g(t, x)$. Gathering constants, the above expressions can be compactly represented as:

$\mu_{r}=\sum_{n=1}^{r} \beta_{n} \phi^{n}$

where the $\beta_{n}$ terms are constants. This polynomial relation presumably holds for all positive $r$, subject to the existence of the $Z$ ratios.

The $Z$ ratios reduce to zero for the special case of purely advective transport because the associated cumulant values are then zero. Therefore, for purely advective transport in the respective stream tubes the central moment expressions are the last terms in Eqs. (16)-(18) and Eq. (19) simplifies to:

$\mu_{r}=\beta_{r} \phi^{r}$

From Eq. 20, the pure advection $g(t, x)$ moment measures of skewness and kurtosis are the constant terms $\beta_{3} / \beta_{2}^{3 / 2}$ and $\beta_{4} / \beta_{2}^{2}$, respectively. This indicates that as $\phi$ increases all $g(t, x)$ distributions will tend toward a stable shape because the last terms in Eqs. (16)-(18) will dominate when $\phi$ is large, leading to a pure advection situation. There is no universal limit $g(t, x)$ shape, however, and different $g(t, x)$ distributions will tend toward different limiting forms. The coefficients of variation of the individual $f_{i}(t, x)$ distributions tend toward zero with increasing travel time (or distance). All limit $g(t, x)$ distributions will be therefore be $N$-modal with probability densities concentrated on $N$ specific time points.

A consequence of Eq. (20) is that for pure advective flow the $\mu_{r} / \phi^{r}$ ratios will be constants independent of travel distance $x$. This has been noted previously in the literature for the special case of $\mu_{2} / \phi^{2}$, which is the square of the coefficient of variation of $g(t, x)$ [24]. However, to the author's knowledge the more general expressions (19) and (20) have not appeared previously in the groundwater literature of contaminant dispersion in parallel flow systems. 
Because $\phi$ is proportional to tracer travel distance $x$, it is evident from Eq. (19) that the $r$ th central moment of $g(t, x)$ varies as an $r$ th order polynomial function of both $x$ and $\phi$. This result is general for perfectly parallel flow systems in that it holds for any $N \geq 2$, for any arbitrary set of $f_{i}(t, x)$ distributions which possess finite moments, and for arbitrary independent advective-dispersive transport in the respective stream tubes.

The variance of $g(t, x)$ is an increasing quadratic function of travel distance because the last term in Eq. (16) is always positive. The latter follows from Jensen's inequality [39, p. 579], noting that

$N^{-1} \sum \alpha_{i}=1$. The situation is more complex for the higher central moments because higher-order polynomials have an increased degree of flexibility when there are possibilities for sign changes in the coefficients. In particular, there may be sign changes in the skewness of $g(t, x)$ for small travel times and distances when $\phi$ is not yet large enough to allow the dominance of the last term in Eq. (17). Similarly, over small travel times and distances there may be fluctuations in the magnitude of the fourth central moment given by Eq. (18), causing kurtosis to change unpredictably.

\section{Discussion}

This paper has been concerned with establishing the distribution-free generality of the polynomial moment relations for parallel flow systems, as opposed to reviewing existing data analyses and field experiments. However, some of the parameterisations given here suggest specific analysis methods for practical use when observed arrival-time variances show a quadratic increase with tracer travel distance. Two potential areas of application are outlined briefly below

\subsection{Estimating the large-distance $g(t, x)$ coefficient of variation}

The coefficient or variation (or its square) has been noted from time to time as a useful quantifier of tracer dispersion characteristics [27, 2, 33]. The coefficient of variation is particularly relevant for parallel systems because it is a distance-invariant constant when $x$ is sufficently large for advective 
effects to dominate, as noted in Section 3. That is, a large value of $x$ implies $\phi$ is large and the first term in Eq. (16) can be neglected, giving the large-distance coefficient of variation as:

$\sqrt{\mu_{2} / \phi^{2}}=\sqrt{N^{-1} \sum \alpha_{i}^{2}-1}$

A simple regression-based estimate of the coefficient of variation (21) is suggested after dividing both sides of Eq. (16) by $\phi$ to give the variance/mean ratio $\mu_{2} / \phi$ as a linear function of $\phi$ :

$\mu_{2} / \phi=N^{-1} \sum Z_{2 i} \alpha_{i}+\phi N^{-1} \sum \alpha_{i}^{2}-1$

where the function gradient corresponds to the square of Eq. (21). An estimate of the large-distance coefficent of variation can therefore be obtained as the square root of the gradient of a linear function fitted through sample estimates of $\mu_{2} / \phi$ plotted against $\phi$.

The estimation procedure is illustrated in Fig 2 by application to two simulated data sets which have been previously noted as exhibiting a quadratic variance increase with travel distance [26]. When plotted as variance/mean ratios against mean travel time, the two data sets both illustrate a high degree of linearity to yield dimensionless least-squares gradient values of 0.503 and 0.133 . The corresponding square root values of 0.709 and 0.365 give the respective estimates of the largedistance coefficients of variation of the respective arrival times.

The required travel distances for these simulated data sets did not in fact have to be very "large" because the evident near-zero vertical intercepts of the fitted linear functions are consistent with the data being derived from purely advective motion in a parallel system. This is also supported by the evident constancy of form of the simulated arrival time distributions in [26]. Interestingly, however, the simulated data was generated using fractional Brownian motion rather than a parallel flow model. The possibility is thus raised that some fractal heterogeneity models may have an approximate mathematical equivalence to pure advective transport in parallel flow systems. Parallel flow models might therefore have wider practical application than just physical settings with evident geological stratification or preferential flow paths. 

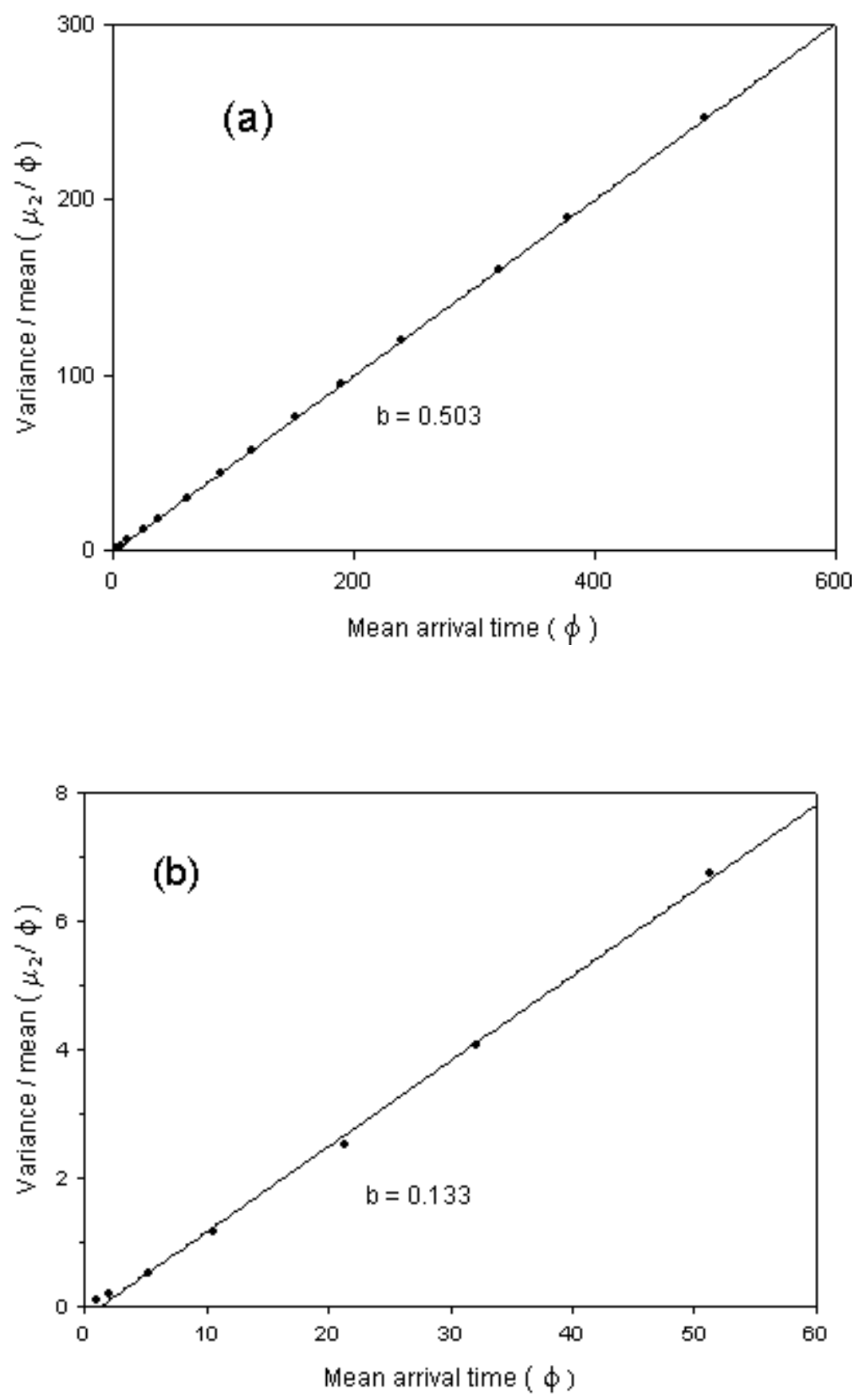

Fig. 2. Plots of variance/mean ratios against mean arrival time for simulated data used by [26]. The $b$ values are the gradients of the best fit lines and (a) and (b) respectively correspond to the data sets designated L=400 and L=50 in Fig. 5 of [26]. All quantities are in arbitrary units. 


\subsection{Modeling arrival-time distributions}

The finite-mixture model given by Eq. (1) provided a useful starting point for developing the polynomial moment relations presented here. However, a suitably parameterised version of (1) could also serve as a model in itself for describing the evolving form of complete $g(t, x)$ distributions. This may be particularly useful for the case where $g(t, x)$ is multimodal, which restricts the amount of distributional information contained in the first few moments [14]. As noted earlier, a feature of distributions related to Eq. (1) is that there will be an increasing tendency toward multimodality of $g(t, x)$ as $x$ increases, with an $N$-mode limit distribution as $x \rightarrow \infty$. Multiple modes of $g(t, x)$ will become evident most rapidly when both $N$ and the $f_{i}(t, x)$ coefficients of variation are small. For example, $g(t, x)$ bimodality has been demonstrated from numerical simulations with $N=2$ [10].

This section describes a specific mixed distribution model which may be useful as a descriptor for the distance evolution of (possibly multimodal) arrival-time distributions with evident quadratic variance increase with travel distance $x$.

Converting Eq. (1) to an expression for practical application requires specification of both $N$ and the component $f_{i}(t, x)$ distributions. Also, the practicalities of having $N$ no larger than necessary requires that the equal weighting in Eq. (1) be replaced with unequal weights. It is well known that the $f_{i}(t, x)$ distributions tend toward normal distributions for homogeneous advective-dispersive transport over large distances [3], suggesting that a potentially useful $g(t, x)$ finite-mixture model could be created from $N$ different normal $f_{i}(t, x)$ distributions with different associated weights. In keeping with the advective-dispersive process, the $f_{i}(t, x)$ distributions should be parameterised such that their respective means and variances increase linearly with travel distance. Lumping parameters and noting the constancy of the $f_{i}(t, x)$ variance/mean ratios $Z_{2 i}$, a normal finite-mixture model for application to advective-dispersive arrival-time data can be written:

$$
h(t, x)=\sum_{i=1}^{N}\left(Z_{2 i} a_{i} x\right)^{-1 / 2} \exp \left[-\frac{\left(t-a_{i} x\right)^{2}}{2 Z_{2 i} a_{i} x}\right] w_{i} \quad w_{i}, a_{i}, Z_{2 i}>0
$$


where $a_{i} x=\mu_{i}$. The weights $w_{i}$ need not sum to unity in this case because they incorporate a scale parameter relating to the units of tracer concentration measurement. The interpretation of the $w_{i}$ values is that they are proportional to the amount of tracer transported in each stream tube.

A suitable value of $N$ would have to be determined by trial and error. However, it is likely that different values of $N$ could serve equally well in many situations. For a given value of $N$, fitting Eq. (23) to arrival-time data will require non-linear estimation of a total of $3 N$ parameter values, consisting of equal numbers of $Z_{2 i}, a_{i}$, and $w_{i}$ terms. This can be specified in a least-squares sense as finding the $3 N$ parameter values which minimise:

$\sum_{m=1}^{M} \sum_{v=1}^{V(m)}\left[h\left(t_{m, v}, x_{m}\right)-c\left(t_{m, v}, x_{m}\right)\right]^{2}$

where $M$ represents the number of different arrival-time measurements sites of flux-weighted tracer concentration along the line of travel from source, $V(m)$ is the number of concentration measurements taken over time at the $m$ th measurement site, $x_{m}$ is the distance between the $m$ th site and the tracer input point, $t_{m, v}$ is the time of the vth concentration measurement at the $m$ th site, and $c\left(t_{m, v}, x_{m}\right)$ is the $v$ th concentration measurement at the $m$ th site.

The usual numerical precautions need to be followed to reduce the effect of multiple solutions when minimising Eq. (24) using some suitable routine. In particular, upper bounds should be placed on all $Z_{2 i}$ values to ensure that a zero weighting is achieved by the $w_{i}$ value concerned going to zero, rather than the associated $Z_{2 i}$ value becoming very large. Also there should be a prior specification of a rank order for all the $a_{i}$ values with some fixed minimum small difference between consecutive $a_{i}$ values.

Fig. 3 shows an example application of fitting the normal mixture model given by Eq. (23). The synthetic tracer concentration data (Table 1) was generated for three different travel distances using a 
two-component inverse Gaussian finite mixture distribution parameterised to have variance increasing as a quadratic function of travel distance. Both inverse Gaussian component distributions were given a skewed form to avoid fortuitous fitting using the normal mixture model. Six normal distributions were utilised, creating a total of 18 unknown parameters to be estimated from the fitting process. It is possible that an $N$ value less than six could serve equally well in this example.

\begin{tabular}{|l|l|l|l|l|l|}
\hline$t$ & $\mathrm{C}(\mathrm{t}, 30)$ & $\mathrm{t}$ & $\mathrm{C}(\mathrm{t}, 50)$ & $\mathrm{t}$ & $\mathrm{C}(\mathrm{t}, 100)$ \\
\hline 1 & 0.009 & 3 & 0.010 & 1 & 0.000 \\
\hline 3 & 0.635 & 5 & 0.123 & 10 & 0.003 \\
\hline 4 & 0.850 & 8 & 0.364 & 20 & 0.107 \\
\hline 6 & 0.926 & 14 & 0.508 & 30 & 0.234 \\
\hline 8 & 0.828 & 17 & 0.478 & 40 & 0.265 \\
\hline 12 & 0.587 & 20 & 0.430 & 50 & 0.236 \\
\hline 20 & 0.295 & 30 & 0.269 & 60 & 0.190 \\
\hline 30 & 0.151 & 40 & 0.163 & 80 & 0.108 \\
\hline 35 & 0.137 & 50 & 0.101 & 100 & 0.057 \\
\hline 40 & 0.151 & 60 & 0.071 & 120 & 0.029 \\
\hline 50 & 0.191 & 80 & 0.110 & 140 & 0.020 \\
\hline 60 & 0.170 & 90 & 0.132 & 170 & 0.058 \\
\hline 70 & 0.112 & 100 & 0.124 & 200 & 0.082 \\
\hline 80 & 0.060 & 120 & 0.062 & 220 & 0.056 \\
\hline 90 & 0.029 & 140 & 0.019 & 250 & 0.017 \\
\hline 100 & 0.013 & 170 & 0.002 & 300 & 0.001 \\
\hline
\end{tabular}

Table 1. Simulated data: tracer concentration arrival times at distances of 30, 50, and 100 units from tracer release point.

Initial parameter estimates were obtained by manually adjusting the parameters for each distribution in turn to obtain an approximate fit of Eq. (23) to the combined data set of $x=30$ and $x=50$. The Excel Solver routine was then used to find a minimum of Eq. 24 which yielded the 18 fitted parameter estimates listed in Table 2. The data for $x=100$ was not used in the fitting process. Fig 3 $(a, b)$ indicates that a good data fit was achieved, as expected from the flexibility of the multiparameter normal mixture. It is probable that the highly non-linear nature of normal mixtures will give rise to different parameter combinations which could fit the same data equally well. Although 
individual parameter values may thus have little meaning, the fitting process could still provide a pragmatic tool for $g(t, x)$ forecasting.
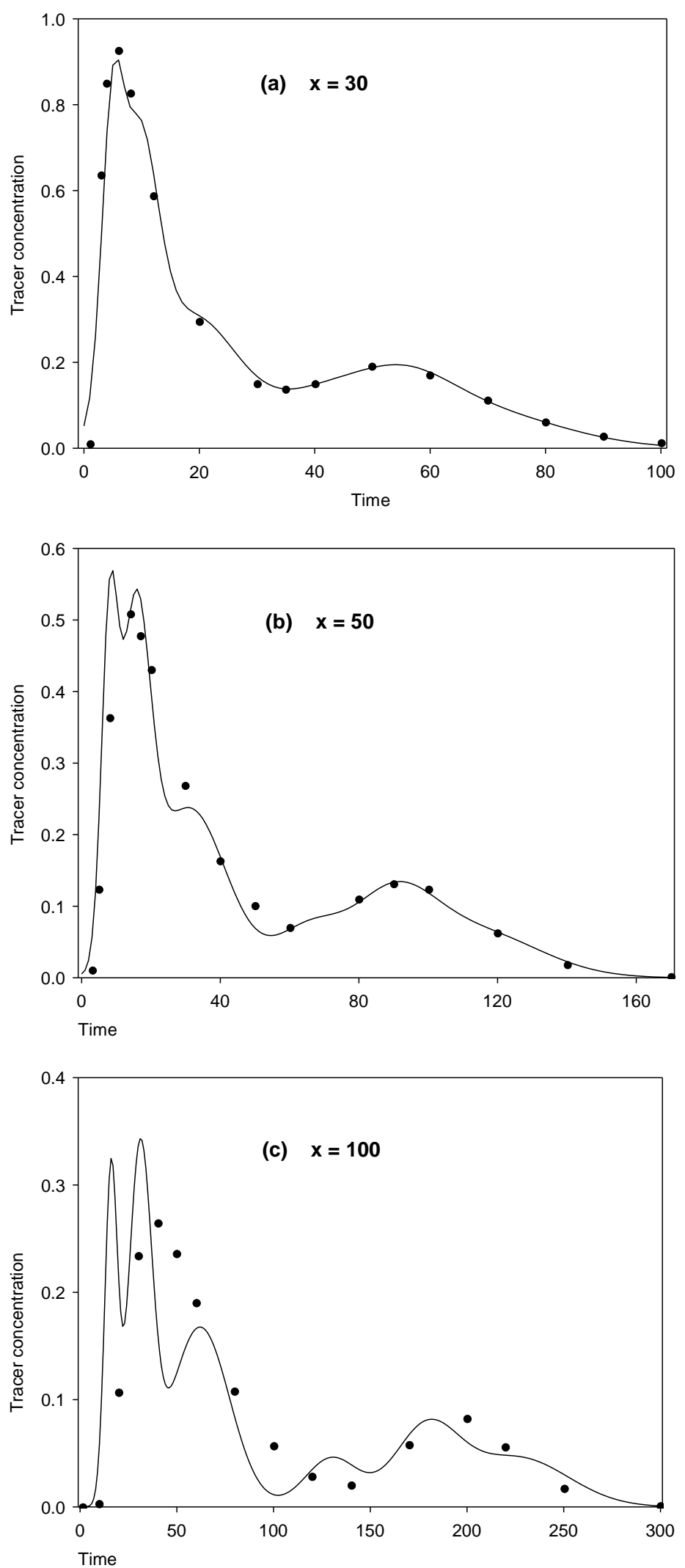

Fig. 3 Fitted and forecast tracer arrival-times using simulated data (solid points). The continuous lines in (a) and (b) are from a collective fit of Eq. (23) to these two data sets. The resulting fitted parameter values (Table 2) give the tracer concentration forecast in (c) when $x$ is set to 100 . 


\begin{tabular}{|l|l|l|l|l|l|l|}
\hline Distribution: & 1 & 2 & 3 & 4 & 5 & 6 \\
\hline $\mathrm{a}_{i}$ & 0.16 & 0.31 & 0.62 & 1.30 & 1.79 & 2.26 \\
\hline $\mathrm{Z}_{2, i}$ & 0.67 & 1.17 & 3.73 & 1.44 & 1.55 & 3.07 \\
\hline$w_{i}$ & 1.01 & 1.94 & 2.55 & 0.62 & 1.19 & 1.21 \\
\hline
\end{tabular}

Table 2 Optimised values of the 18 parameters obtained from fitting Eq. 23 (for $N=6$ ) to the combined data of Table 1 for $x=30$ and $x=50$.

An example forecast is shown as the continuous line in Fig. 3c, obtained from Eq. 23 using the Table 2 parameter values and setting $x$ to 100 . The general bimodal form of the data is anticipated by the tracer concentration forecast. However, the forecast function is already showing the initial stages of decomposition into its limit 6-mode form. The forecasts here were made on the basis of fitting just two data sets at different distances because it was already known that the simulated data had variance increasing as a quadratic function of travel distance. A minimum of three different tracer recording sites would be required in field experiments to first determine whether the quadratic model is appropriate.

\section{Conclusion}

A simple general polynomial relation provides a useful summary statement of temporal central moment variation as a function of the travel distance of a conservative tracer pulse in a perfectly parallel flow system. That is, the $r$ th temporal central moment was shown to be an $r$ th order polynomial function of tracer travel distance, independent of considerations of arrival-time distributions and hydraulic conductivity variations between stream tubes. The relation confirms the well-known quadratic variance increase for pure advective flow in parallel systems, which is generalised to include arbitrarily different advective-dispersive transport (linear variance increase) in the different stream tubes. The polynomial relations anticipate that advective-dispersive transport processes could cause arrival-time skewness and kurtosis to fluctuate unpredictably near tracer source. However, pure advection plays an increasingly dominant role with increasing travel distance, causing the arrival-time distribution to tend toward some fixed form with distance-invariant skewness and kurtosis. 
While the polynomial relation is general within the framework of the perfectly parallel flow model, the parallel model is itself is a somewhat idealised concept as an approximation to realty. It might be expected, for example, that the inevitable component of transverse mixing will cause the parallel model to break down with increasing travel distance in field situations. However, analysis of parallel systems with partial mixing suggest that these effects impact only slowly with tracer travel distance [12]. A pragmatic check for the parallel model applicability would be to observe whether arrival-time variance increases as an approximately quadratic function of travel distance in a given field situation. Evident non-quadratic variance increases would cause rejection of the parallel model and the associated polynomial moment relations.

Some generalisation of the polynomial moment model should be possible as part of further development. In particular, it would be interesting to establish whether polynomial expressions also apply for the temporal moments of reactive tracers when combined with arbitrary tracer input rates over some specified time duration.

\section{Acknowledgement}

The simulated data plotted in Fig. 2 was kindly provided by Scott Painter, Center for Nuclear Waste Regulatory Analyses, Southwest Research Institute, San Antonio, Texas. The development of this paper was enhanced by useful comments in the review process. 


\section{References}

[1] Aris R. On the dispersion of linear kinematic waves. Proc R Soc London Ser A 1958;245:268-77.

[2] Buffham BA, Mason G. Holdup and dispersion: tracer residence times, moments and inventory measurements. Chem Eng Sci 1993; 48:3879-887.

[3] Chatwin PC. The approach to normality of the concentration distribution of solute in a solvent flowing along a straight pipe. J Fluid Mech 1970;51:321-52.

[4] Chen CT, Li SH. Radionuclide transport in fractured porous-media - analytical solutions for a system of parallel fractures with a flux-type boundary-condition. Nucl Technol 1997;117:223-33.

[5] Cirpka OA, Kitanidis PK. An advective-dispersive stream tube approach for the transfer of conservative-tracer data to reactive transport. Water Resour Res 2000;36:1209-220.

[6] Cirpka OA, Kitanidis PK. Characterization of mixing and dilution in heterogeneous aquifers by means of local temporal moments. Water Resour Res 2000;36:1221-236.

[7] Cvetkovic VD, Shapiro AM. Solute transport in stratified formations. Water Resour Res $1989 ; 25: 1283-289$.

[8] Cvetkovic V, Shapiro AM, Dagan G. A solute flux approach to transport in heterogeneous formations 2. Uncertainty analysis. Water Resour Res 1992;28:1377-388.

[9] Daniel MM, Willsky AS, McLaughlin D. A multiscale approach for estimating solute travel time distributions. Adv Water Resour 2000;23:653-65.

[10] Desbarats AJ. Macrodispersion in sand-shale sequences. Water Resour Res 1990;26:153-63. 
[11] Demmy G, Berglund S, Graham W. Injection mode implications for solute transport in porousmedia - analysis in a stochastic Lagrangian framework. Water Resour Res 1999;35:1965-973.

[12] Gelhar LW, Gutjahr AL, Naff RL. Stochastic aquifer analysis of macrodispersion in a stratified aquifer. Water Resour Res 1979;15:1387-397.

[13] Güven O, Molz, FJ, Melville JG. An analysis of dispersion in a stratified aquifer. Water Resour Res 1984;20:1337-354.

[14] Harvey CF, Gorelick SM. Temporal moment-generating equations: modelling transport and mass transfer in heterogeneous aquifers. Water Resour Res 1995;31:1895-911.

[15] Herrling B, Leismann HM. Modelling the increasing dispersivity with FE transport models using the multilayer concept. In: Custodio E, Gurgui A, Lobo Ferreira JP. Groundwater flow and quality modelling. Boston, USA; Reidel; 1988. p. 463-74.

[16] Jury WA, Roth K. Transfer functions and solute movement through soil. Boston, USA: Birkhauser; 1990.

[17] Keum DK, Park CK, Hahn PS, Vandergraaf TT. A stratified channel model with local longitudinal dispersion. Nucl Technol 1997;120:211-23.

[18] Li SG, McLaughlin D. Asymptotic properties of the Eulerian truncation approximation: analysis of the perfectly stratified transport problem. Water Resour Res 2002;38 8 10.1029/2000WR000040.

[19] Marle C, Simandoux P, Pacsirsky J, Gaulier C. Etude du déplacement de fluides miscibles en milieu poreaux stratifié. Rev Inst Francais Pétrol 1967;22:272-94.

[20] Matheron G, de Marsily G. Is transport in porous media always diffusive? A counterexample. Water Resour Res 1980;16:901-17. 
[21] Mercado A. The spreading pattern of injected water in a permeability stratified aquifer. In: Symposium of Haifa, Artificial Recharge and Management of Aquifers, IAHS Publ. 72, Gent Brugge, Belgium, 1967. p. 23-36.

[22] Naff RL. Arrival times and temporal moments of breakthrough curves for an imperfectly stratified aquifer. Water Resour Res 1992;28:53-68.

[23] Nelson RW. Evaluating the environmental consequences of groundwater contamination 1. An overview of contaminant arrival distributions as general evaluation requirements. Water Resour Res 1978;14:409-15.

[24] Neretnieks I. A note on fracture flow dispersion mechanisms in the ground. Water Resour Res 1983;19:364-70.

[25] Oya S, Valocchi AJ. Transport and biodegradation of solutes in stratified aquifers under enhanced in-situ bioremediation conditions. Water Resour Res 1998;34:3323-334.

[26] Painter S, Mahinthakumar G. Prediction uncertainty for tracer migration in random heterogeneities with multifractal character. Adv Water Resour 1999;23:49-57.

[27] Pannone M, Kitanidis PK. Large-time behavior of concentration variance and dilution in heterogeneous formations. Water Resour Res 1999;35:623-34.

[28] Pickens JF, Grisak GE. Scale-dependent dispersion in a stratified granular aquifer. Water Resour Res 1981;17:1191-211.

[29] Rao PV,. Portier KM, Rao PSC. A stochastic approach for describing convective-dispersive solute transport in saturated porous media. Water Resour Res 1981;17:963-68.

[30] Rasmuson A. Analysis of hydrodynamic dispersion in discrete aquifer networks using the method of moments. Water Resour Res 1985;21:1677-683. 
[31] Rubin Y, Ezzedine S. The travel times of solutes at the Cape Cod tracer experiment: data analysis, modeling, and structural parameters inference. Water Resour Res 1997;33:1537-547.

[32] Shapiro AM, Cvetkovic VD. Stochastic analysis of solute arrival time in heterogeneous porous media. Water Resour Res 1988;24:1711-718.

[33] Simmons CS. A stochastic-convective transport representation of dispersion in one-dimensional porous media systems. Water Resour Res 1982;18:1193-214.

[34] Stuart A, Ord JK, Kendall's advanced theory of statistics, v1. 5th Ed. London: Griffin; 1987.

[35] Thorbjarnarson KW, Mackay DM. A field-test of tracer transport and organic contaminant elution in a stratified aquifer at the Rocky-Mountain Arsenal (Denver, Colorado, USA). J Contam Hydr 1997;24:287-312.

[36] Woodbury AD, Rubin Y. A full-Bayesian approach to parameter inference from tracer traveltime moments and investigation of scale effects at the Cape-Cod experimental site. Water Resour Res 2000;36:159-71.

[37] Young DF, Ball WP. Column experimental design requirements for estimating model parameters from temporal moments under nonequilibrium conditions. Adv Water Resour 2000;23:449-60.

[38] Yu C, Warrick AW, Conklin MH. A moment method for analyzing breakthrough curves of step inputs. Water Resour Res 1999;35:3567-572.

[39] Zwillinger D. Ed. CRC standard mathematical tables and formulae. 30th Ed. New York: Chemical Rubber Company; 1996. 